Gynäkologe 2016 • 49:884

DOI 10.1007/s00129-016-3964-4

Online publiziert: 5. Oktober 2016

๑) Springer-Verlag Berlin Heidelberg 2016

CrossMark
J. Wilm · S. Schüler-Toprak · 0. Ortmann

Caritas Krankenhaus St. Josef, Universitätsfrauenklinik Regensburg, Regensburg, Deutschland

\section{Erratum zu: Krebsfrüherkennung des Zervix- und des Mammakarzinoms}

Erratum zu:

Gynäkologe (2016) 49(7):535-548

DOI 10.1007/s00129-016-3907-0

In diesem Beitrag ist die Angabe über die jährliche Tastuntersuchung für Brustkrebs in - Tab. 1 fehlerhaft. Die Tastuntersuchung wird erst ab 30 Jahren von den gesetzlichen Kassen übernommen. Wir bitten, die im Folgenden korrekt dargestellte Tabelle zu beachten.

Die Autoren bitten, den Fehler zu entschuldigen.

\section{Korrespondenzadresse}

\section{J. Wilm}

Caritas Krankenhaus St. Josef, Universitätsfrauenklinik Regensburg

Landshuter Straße 65, 93053 Regensburg,

Deutschland

jwilm@caritasstjosef.de

Tab. 1 Krebsfrüherkennungsmaßnahmen, die bei Frauen durchgeführt werden und deren Kosten von den gesetzlichen Krankenkassen übernommen werden. (Mod. nach [5])

\begin{tabular}{|c|c|c|c|}
\hline Erkrankung & $\begin{array}{l}\text { Anspruchsalter } \\
\text { (Jahre) }\end{array}$ & Intervall & Erläuterung \\
\hline \multirow{3}{*}{$\begin{array}{l}\text { Gebärmutterhalskrebs } \\
\text { (geplantes Vorgehen ab } \\
\text { 2018) }\end{array}$} & Ab 20 & Jährlich & Gynäkologische Untersuchung \\
\hline & Ab 20 bis 30 & Jährlich & Pap-Abstrich \\
\hline & Ab 30 Jahren & Alle 5 Jahre & $\begin{array}{l}\text { HPV-Test, alternativ jährlich } \\
\text { Pap-Abstrich }\end{array}$ \\
\hline \multirow[t]{2}{*}{ Brustkrebs } & $\mathrm{Ab} 30$ & Jährlich & Tastuntersuchung \\
\hline & Ab 50 bis 69 & Alle 2 Jahre & Mammographie \\
\hline Hautkrebs & $\mathrm{Ab} 35$ & Alle 2 Jahre & Hautinspektion \\
\hline \multirow[t]{2}{*}{ Darmkrebs } & $\mathrm{Ab} 50$ & Jährlich & Test auf okkultes Blut im Stuhl \\
\hline & $\mathrm{Ab} 55$ & Alle 2 Jahre & $\begin{array}{l}\text { Test auf okkultes Blut im Stuhl, } \\
\text { alternativ max. } 2 \text { Koloskopien im } \\
\text { Abstand von } 10 \text { Jahren }\end{array}$ \\
\hline
\end{tabular}

Die Online-Version des Originalbeitrags finden Sie unter http://dx.doi.org/10.1007/s00129016-3907-0. 